\title{
Identification of a novel bovine enterovirus possessing highly divergent amino acid sequences in capsid protein
}

Shinobu Tsuchiaka 1,2, Sayed Samim Rahpaya ${ }^{1,2}$, Konosuke Otomaru ${ }^{3}$, Hiroshi Aoki ${ }^{4}$, Mai Kishimoto², Yuki Naoi², Tsutomu Omatsu ${ }^{1,2}$, Kaori Sano ${ }^{2}$, Sachiko Okazaki-Terashima ${ }^{1,2}$, Yukie Katayama², Mami Oba ${ }^{2}$, Makoto Nagai ${ }^{5}$ and Tetsuya Mizutani ${ }^{1,2^{*}}$

\begin{abstract}
Background: Bovine enterovirus (BEV) belongs to the species Enterovirus E or F, genus Enterovirus and family Picornaviridae. Although numerous studies have identified BEVs in the feces of cattle with diarrhea, the pathogenicity of BEVs remains unclear. Previously, we reported the detection of novel kobu-like virus in calf feces, by metagenomics analysis. In the present study, we identified a novel BEV in diarrheal feces collected for that survey. Complete genome sequences were determined by deep sequencing in feces. Secondary RNA structure analysis of the $5^{\prime}$ untranslated region (UTR), phylogenetic tree construction and pairwise identity analysis were conducted.
\end{abstract}

Results: The complete genome sequences of BEV were genetically distant from other EVs and the VP1 coding region contained novel and unique amino acid sequences. We named this strain as BEV AN12/Bos taurus/JPN/2014 (referred to as BEV-AN12). According to genome analysis, the genome length of this virus is 7414 nucleotides excluding the poly (A) tail and its genome consists of a $5^{\prime} U T R$, open reading frame encoding a single polyprotein, and $3^{\prime} U T R$. The results of secondary RNA structure analysis showed that in the 5'UTR, BEV-AN12 had an additional clover leaf structure and small stem loop structure, similarly to other BEVs. In pairwise identity analysis, BEV-AN12 showed high amino acid (aa) identities to Enterovirus F in the polyprotein, P2 and P3 regions (aa identity $\geq 82.4 \%$ ). Therefore, BEV-AN12 is closely related to Enterovirus F. However, aa sequences in the capsid protein regions, particularly the VP1 encoding region, showed significantly low aa identity to other viruses in genus Enterovirus (VP1 aa identity $\leq 58.6 \%$ ). In addition, BEV-AN12 branched separately from Enterovirus $E$ and $F$ in phylogenetic trees based on the aa sequences of P1 and VP1, although it clustered with Enterovirus $F$ in trees based on sequences in the P2 and P3 genome region.

Conclusions: We identified novel BEV possessing highly divergent aa sequences in the VP1 coding region in Japan.

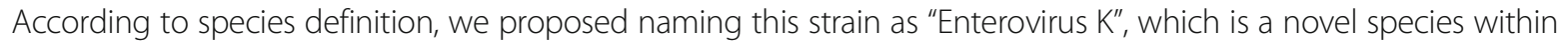
genus Enterovirus. Further genomic studies are needed to understand the pathogenicity of BEVs.

Keywords: Bovine enterovirus, Deep sequencing, Phylogenetic analysis

\footnotetext{
* Correspondence: tmizutan@cc.tuat.ac.jp

${ }^{1}$ The United Graduate School of Veterinary Sciences, Gifu University, 1-1 Yanagito, Gifu-shi, Gifu 501-1193, Japan

${ }^{2}$ Research and Education Center for Prevention of Global Infectious Disease of Animals, Tokyo University of Agriculture and Technology, 3-5-8 Saiwai-cho, Fuchu-shi, Tokyo 183-8509, Japan

Full list of author information is available at the end of the article
} International License (http://creativecommons.org/licenses/by/4.0/), which permits unrestricted use, distribution, and reproduction in any medium, provided you give appropriate credit to the original author(s) and the source, provide a link to the Creative Commons license, and indicate if changes were made. The Creative Commons Public Domain Dedication waiver (http://creativecommons.org/publicdomain/zero/1.0/) applies to the data made available in this article, unless otherwise stated. 


\section{Background}

Bovine enterovirus (BEV) is a single positive-stranded RNA virus belonging to the genus Enterovirus within family Picornaviridae. The viral particle is composed of a small, non-enveloped and icosahedral virion and $7.5 \mathrm{k}-$ base genome containing a single open reading frame (ORF) flanked by untranslated regions (UTRs) at the 5' and 3 ' ends. The ORF encodes a single long polyprotein containing structural proteins (VP1, VP2, VP3 and VP4 encoded in $\mathrm{P} 1$ ) and non-structural proteins (2A, 2B and $2 \mathrm{C}$ encoded in $\mathrm{P} 2$ as well as $3 \mathrm{~A}, 3 \mathrm{~B}, 3 \mathrm{C}$ and $3 \mathrm{D}$ encoded in P3) $[1,2]$.

Genus Enterovirus is divided into 12 species defined as Enterovirus $A-H$ and $J$ (EV-A, B, C, D, E, F, G, H and J) and Rhinovirus $A-C$ (RV-A, B and C) [2]. BEVs belong to EV-E and EV-F (formerly known as BEV-A and BEV$B$, respectively) and can be distinguished from other EVs by the unique secondary structure of their RNA genome: a 5 '-cloverleaf and internal ribosome entry site (IRES) linked by additional nucleotide sequences at the 5 'UTR [3-5]. Since the isolation of BEVs from cattle in the late 1950s [6-8], studies worldwide have detected BEVs not only in cattle but also in other animal species including possums, bottlenose dolphins, camels and alpacas [8-12]. Although BEVs have been classified based on virus antigenicity determined by cross neutralization testing [13-16], the genotype based on the capsid protein (particularly in VP1) amino acid sequences are also used to classify BEVs $[4,10-12,17,18]$. BEVs are classified into 4 sero-/genotypes and 6 sero-/genotypes in EV-E (E1, E2, E3 and E4) and EV-F (F1, F2, F3, F4, F5 and F6), respectively.

Although most EVs cause only mild symptoms, including hand-foot-and-mouth disease, herpangina, pleurodynia and rashes $[19,20]$, some members belonging to the genus Enterovirus can cause severe diseases. The most well known pathogen is poliovirus affecting humans. Poliovirus and some of other EVs, including coxsackie virus and echovirus, can invade the central nervous system causing neurological diseases, including aseptic meningitis, encephalitis and ataxia [21, 22]. In other animals, although porcine teschovirus, formerly classified as porcine enterovirus, can cause a neurological disorder known as Teschen/Talfan disease [23], the pathogenicity of EVs infecting animals are still unclear. In case of cattle, footand-mouth disease virus belonging to the genus Aphthovirus of the family Picornaviridae can cause vesicular diseases leading to a serious economic impact for farmers [24]; the pathogenicity of viruses belonging to the genus Enterorovirus is still unclear. Several reports have claimed that BEVs can cause diarrhea, respiratory diseases, reproductive diseases and infertility in cattle [25-27]; however, BEVs have also been widely detected in asymptomatic cattle and their environment, and experimental infection trials of BEV have failed to produce clinical signs
[28-30]. Therefore, whether BEV infection is clinically important remains unclear.

It is widely known that most viruses belonging to genus Enterovirus utilize "canyon" as their binding site to cells surface receptors, which is formed by outer capsid proteins including VP1, VP2 and VP3 [31]. Several studies of other enteroviruses revealed that sequences of the VP1 coding region are responsible for the phenotype of viruses; some amino acid substitutions in this region altered the pathogenicity and cell tropism of the viruses [32-34]. Although the cell surface receptor to BEV has not been identified, it is likely that the capsid proteins, including VP1, may be responsible for the phenotype of BEVs, as their capsid proteins also form a "canyon" on the outer side of the virion, and a strain isolated from cattle with severe symptoms contained specific amino acid substitutions in the capsid regions [27, 35]. To elucidate the determinants of BEV virulence in hosts, genomic information of BEVs must be determined.

Recently, deep sequencing techniques using highthroughput sequencers have been used to evaluate virome including novel viruses in clinical samples without viral isolation to determine total genomic information within samples [36, 37]. We previously identified novel viruses infecting the intestinal tracts of livestock using high-throughput sequencers to study enterovirus, picornavirus and astrovirus in the feces of goat, swine and cattle, respectively [38-41].

Previously, we reported the detection of novel kobulike virus in Japanese Black cattle, using feces of calf, by metagenomics analysis. In the present study, we identified a novel BEV in feces collected for that survey [42]. To characterize the genomic features of this virus, complete genome sequences were determined and phylogenetic trees were constructed. In addition, secondary RNA structures in the 5'UTR and pairwise identity were analyzed.

\section{Methods}

Fecal sample and virus isolation

Previously, we reported the detection of a novel kobulike virus in Japanese black cattle by deep sequencing method [42]. During the metagenomics surveillance, nucleotide sequences with high similarity to BEVs were identified in feces collected from a calf with diarrhea. This feces was collected from a 1-month-old calf with diarrhea in Kagoshima prefecture (Kagoshima sample) in 2014. No other clinical sign was observed except diarrhea. Feces was collected directly from the rectum on the onset day. One gram feces was diluted with $9 \mathrm{~mL}$ PBS (-) to prepare a $10 \%$ fecal suspension and centrifuged at $10,000 \times \mathrm{g}$ for $10 \mathrm{~min}$. The supernatant was collected and stored at $-80{ }^{\circ} \mathrm{C}$ before RNA extraction and virus isolation. 
The supernatant of the Kagoshima sample was subjected to virus isolation. The fecal supernatant was filtered through a $0.45-\mu \mathrm{m}$ pore size membrane and treated with $10 \mu \mathrm{g} / \mathrm{mL}$ acetylated trypsin (Sigma-Aldrich, St. Louis, MO, USA) for $60 \mathrm{~min}$ at room temperature before virus isolation. Treated samples were inoculated into Mardin-Darby bovine kidney cells. Blind passage was subsequently conducted three times. Minimum Essential Medium was used as negative control (Sigma-Aldrich).

\section{Isolated BEV strains}

In this study, three BEVs isolated in Japan, BEV IS1/Bos taurus/JPN/1990 (BEV-IS1) and IS2/Bos taurus/JPN/ 1990 (BEV-IS2), were additionally sequenced and analyzed. These viruses were isolated from a fecal sample collected from one cow at the same time in 1990 in the Ishikawa prefecture (The clinical features of cattle infected with BEV-IS1 and IS2 have not been recorded). In addition, BEV Ho12/Bos taurus/JPN/2009 (BEV-Ho12) was isolated from diarrheic feces collected in Hokkaido in 2009 by as described above [39].

\section{RNA extraction, cDNA library construction and whole genome sequencing}

Total RNA was extracted from $0.25-\mathrm{mL}$ supernatants of isolated viruses and $10 \%$ fecal samples using TRIzol LS Reagent (Life Technologies, Carlsbad, CA, USA). RNA samples were normalized to $10-100 \mathrm{ng}$ of RNA per reaction, using a Qubit_2.0 Fluorometer (Invitrogen, Carlsbad, CA, USA). The cDNA library of sample RNA was constructed using the NEBNext Ultra RNA Library Prep Kit for Illumina version 2.0 (New England Biolabs, Ipswich, MA, USA) as described previously [40] and sequenced using MiSeq (Illumina, San Diego, CA, USA) with the MiSeq reagent kit V2 (300 cycles) (Illumina). Briefly, all reads were generated as 151 paired end reads. Each sample was multiplexed with other 23 samples prepared from diarrheal feces of other calves (data not shown). 5'-Full RACE Core Set (TaKaRa Bio, Shiga, Japan) and 3'Full RACE Core Set (TaKaRa Bio) were used to complement virus sequences of the $5^{\prime}$ end and 3 ' end, respectively.

\section{Analysis of genome sequences}

All nucleotide sequences determined by Miseq (referred to as "reads") were converted to FASTAQ format on MiSeq reporter V2.3 and subsequently analyzed using CLC Genomics Workbench 6.0 (CLC bio, Cambridge, MA, USA). Briefly, the ends of all reads were trimmed to remove adaptor sequences located at both ends of each read. Trimmed reads were assembled into contigs using a de novo assembly algorithm. Contigs generated by de novo assembly algorithm were analyzed using BlastN.
Hypothetical polyprotein cleavage sites of the viruses were predicted by multiple alignments with other BEVs and confirmed by the NetPicoRNA [43]. Nucleotide (nt) sequences or amino acid (aa) sequences were aligned using ClustalW. Phylogenetic trees were constructed by maximum likelihood (ML) methods on MEGA5.2.2 [44]. The mtREV24 + G + F model (5'UTR), rtREV + F model (3'UTR), rtREV + G + F model (P1), rtREV + G + I (P2 and P3), and WAG + G + I (VP1) were employed as evolutionary models for ML method. Pairwise identity was analyzed on CLC Genomics Workbench and the secondary RNA structure of the 5'UTR was predicted by Mfold [45].

\section{VP1 genome sequencing}

RT-PCR was performed by using PrimeScript One Step RT-PCR Kit Ver.2 (TaKaRa Bio) to confirm the sequences of the contigs obtained from the Kagoshima sample. Three primer sets were designed based on the contig sequences of this sample. Primer sequences are given in Additional file 1: Table S1. PCR products were sequenced using a 3130xl Genetic analyzer (Applied Biosystems, Foster City, CA, USA).

\section{Detection of other pathogens causing diarrhea}

To confirm the presence of other pathogens in the Kagoshima sample, detection of agents causing diarrhea using our real-time PCR system, referred to as "Dembo-PCR," was performed [46]. This system can identify 19 species of pathogens, including virus, bacteria and protozoa. Briefly, viral DNA and RNA were extracted by high pure viral nucleic acid extraction kit (Roche Diagnostics $\mathrm{GmbH}$, Mannheim, Germany) and bacteria and protozoa DNA were extracted by QIAamp Fast DNA stool mini kit (QIAGEN, Hilden, Germany). Nucleic acids extracted by each kit were subjected to Dembo-PCR, according to a previous report [46].

\section{Results \\ Virus isolation and determination of viral genome sequences}

Although virus isolation using supernatants of the Kagoshima feces was repeated three times, no cytopathic effect could be detected. Therefore, RNA extracted from the Kagoshima sample collected in 2014 and virus stocks of BEV-IS1, BEV-IS2 and BEV-Ho12 were subjected to deep sequencing. The Kagoshima sample was sequenced twice, and all reads obtained from the two runs were used to generate contigs (the first and second deep sequencing yielded 1,304,032 and 929,976 reads, respectively). The results of BlastN analysis revealed that bovine enterovirus F, group A rotavirus (RVA), bovine kobu-like virus and bovine picornavirus were identified with $\mathrm{E}$ value $=0$. However, RVA was not detected in feces by Dembo-PCR. 
The total BEV read counts (percentages indicate BEV reads per total reads of the first and second deep sequencing) of the Kagoshima sample were 1202 reads $(0.05 \%)$, and an approximately $7400 \mathrm{nt}$ contig was obtained from the integrated result with a 24.24 average sequence read depth (maximum read depth was 46). The complete genome was determined using $5^{\prime}$ and $3^{\prime}$ end RACE methods. Because amino acid sequences of VP1 were not similar to those of other enteroviruses by homology analysis as described below, the VP1 genome sequence was confirmed by directly sequencing the PCR product. As a result, sequences obtained from direct sequencing agreed with the results of deep sequencing. The genome length of BEV from the Kagoshima sample was $7414 \mathrm{nt}$, excluding the poly (A) tail. We named this $\mathrm{BEV}$ as BEV AN12/Bos taurus/JPN/2014 (BEV-AN12).

Viral genomes of isolated viruses including complete ORFs were also determined. The genome lengths of BEVIS1, BEV-IS2 and BEV-Ho12 were 7413 nt (P1: 2517 nt, P2: $1737 \mathrm{nt}$, and P3: $2271 \mathrm{nt}), 7394 \mathrm{nt}$ (P1: $2496 \mathrm{nt}, \mathrm{P} 2$ : $1734 \mathrm{nt}$, and P3: $2271 \mathrm{nt}$ ), and $7350 \mathrm{nt}$ (P1: $2496 \mathrm{nt}, \mathrm{P} 2$ : $1734 \mathrm{nt}$, and P3: $2271 \mathrm{nt}$ ), respectively. The sequences of BEV-AN12, BEV-Ho12, BEV-IS1 and BEV-IS2 were deposited in the DDBJ/EMBL/GenBank database under the accession numbers LC038188, LC150008, LC150009 and LC150010, respectively.

\section{Pairwise identity and genome analysis}

Table 1 shows the pairwise aa (polyprotein, $2 \mathrm{C}+3 \mathrm{CD}$, P1P3, VP1-VP4 and 3D) or nt (5'UTR and 3'UTR) identity of BEV-AN12 to representative strains of each species belonging to BEVs and other Japanese BEVs. Deduced aa sequences encoding polyprotein, $2 \mathrm{C}+3 \mathrm{CD}, \mathrm{P} 1, \mathrm{P} 2, \mathrm{P} 3,3 \mathrm{D}$ and four capsid proteins (encoding VP4, VP2, VP3 and VP1) were compared to each EV-E and F. BEV-AN12 possessed showed identity to EV-Fs in polyprotein, $2 \mathrm{C}+3 \mathrm{CD}$, P2, P3 and 3D than to those of EV-Es. However, low aa identity (aa identity $<70 \%$ ) was observed in P1 to EV-Es and EV-Fs. Particularly, the VP1 region of BEV-AN12 encoded in P1 showed a significantly low aa identity to other BEVs $(54.7 \% \leq$ aa identity $\leq 58.6 \%)$. As a result of multiple alignment analysis, several motifs conserved among the genomes of genus Enterovirus were detected in the genome of BEV-AN12. In particular, the [PS] ALXAAXETG motif in VP1, GXCG motif in 2A, GXXGXGKS motif for NTP-binding in 2C, GXCG motif forming part of the catalytic active site in $3 \mathrm{C}$, and $\mathrm{KDE}$ [LI] $\mathrm{R}$ in 3D were identified [47-50]. However, the putative cleavage site at the junction of VP3/VP1 was a glutamine/serine for BEV-AN12. In addition, a 6-aa insertion in the 2A region was identified at position $835-840$ aa (PLRTTG) in the BEV-AN12 genome. Multiple alignment using aa sequences encoding polyprotein is supplemented as Additional file 2: Table S2.

\section{Secondary RNA structure of 5'UTR}

The putative secondary 5'UTR RNA structure of BEVAN12 is shown in Fig. 1. Zell et al. reported that the 5' UTR of BEVs form additional cloverleaf structures and small stem loops between the 5 '-cloverleaf structure and IRES, which clearly distinguish BEVs from other EVs [3]. Similarly, our analysis revealed that all Japanese BEVs had BEV-specific structures (domains $\mathrm{I}^{*}$ and $\mathrm{I}^{* *}$ ). Domains II, III, IV, V and VI, which are the main domains of type 1 IRES directing cap-independent translation [51], were also observed in all Japanese BEVs.

\section{Phylogenetic analysis}

Phylogenetic trees based on the nt sequences of $5{ }^{\prime}$ UTR and 3'UTR and aa sequences of P1, P2, P3 and VP1 are shown in Fig. 2 and Fig. 3, respectively. Phylogenetic analysis showed that all Japanese strains of BEV including BEV-AN12 formed a cluster with other BEVs in all six trees. However, BEV-AN12 was completely separated from other BEVs in the P1 and VP1 trees.

\section{Detection of agents causing diarrhea by Dembo-PCR}

Dembo-PCR was performed to identify agents causing diarrhea in calf. According to the results, only BEV was detected by this test. Genome of other pathogens was not identified in the fecal sample of Kagoshima.

\section{Discussion}

According to the species demarcation criteria for the genus Enterovirus defined by the International Committee on Taxonomy of Viruses, members of a species in the genus Enterovirus should share high aa identity (aa $>70 \%$ in the polyprotein, aa $>60 \%$ in P1 and $>80 \%$ aa identity in $2 \mathrm{C}+3 \mathrm{CD}$ ) and compatibility in processing, replication and encapsidation [2]. In addition, EV-E and F can be distinguished from other EVs because of their unique secondary RNA structures in the 5'UTR region (domains I* and I**) [4]. Our genome analysis revealed that BEVAN12 shared aa sequences and protease cleavage site positions with EV-Fs. In addition, BEV-AN12 contained domains $I^{*}$ and $I^{*}$ in the $5^{\prime}$ UTR similarly to other BEVs. Therefore, BEV-AN12 is closely related to EV-Fs. However, pairwise identity analysis revealed that aa sequences in the VP1 region of the BEV-AN12 genome had significantly low identities to other BEVs strains (VP1 aa identity $\leq 58.6 \%$ ). Furthermore, BEV-AN12 did not cluster with any other EV-E and EV-F in the VP1 phylogenetic tree, although its $\mathrm{P} 2$ and $\mathrm{P} 3$ regions were closely related to EV-F. The percentage of aa identity of VP1 is commonly utilized for species and sero-/genotype definition (range from 50 to $55 \%$ for heterologous species, 70 to $85 \%$ for heterologous sero-/genotypes/homologous species, and greater than $90 \%$ for homologous sero-/genotypes) [4]. According to the classification definition, our results 


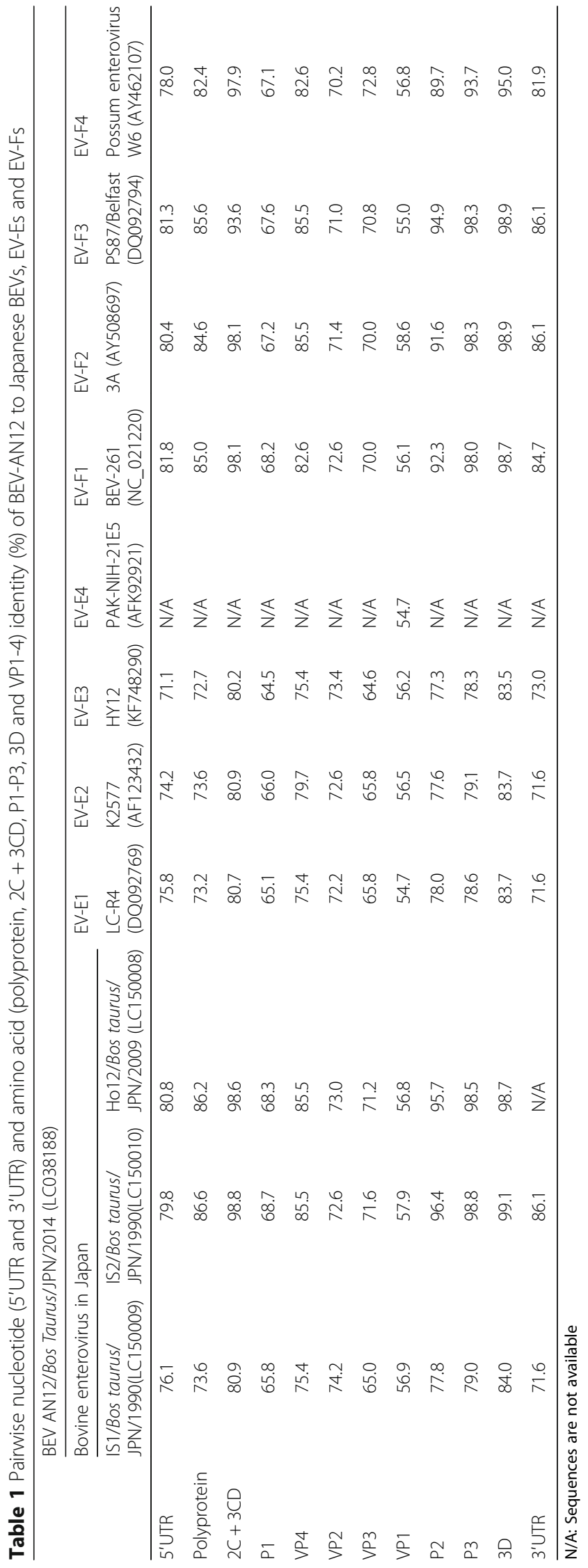




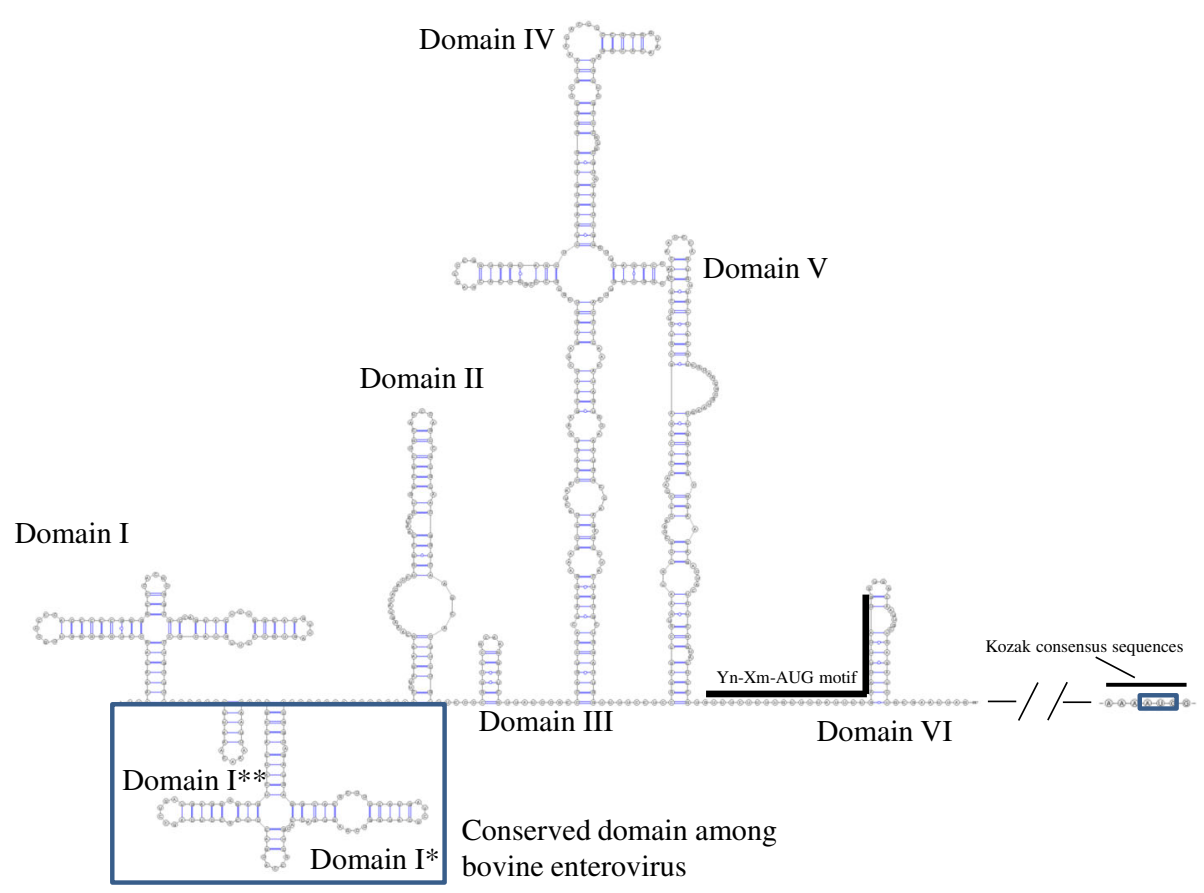

Fig. 1 Secondary RNA structure of 5'UTR of BEV-AN12. Putative secondary RNA structure of 5'UTR of BEV-AN12 was predicted by Mfold. Domains $I, I^{*}, I^{* *}, I I, I I I, I V, V$ and $V I$ domain were predicted. Domains $I^{*}$ and $I^{* *}$ conserved among BEVs are shown with a bold line. Yn-Xm-AUG motif conserved in domain $\mathrm{VI}$ is indicated by a bold line. Kozak consensus sequences with start codon are also indicated by a bold line

indicate that BEV-AN12 is taxonomically distant from previously reported BEVs. Therefore, we named this strain as "Enterovirus $K$ ", which is a novel species within the genus Enterovirus. Other Japanese BEVs were classified as typical BEVs (BEV-IS1: EV-E2, BEV-IS2 and BEV-Ho12: EV-F4).

Our recombination analysis could not reveal the source of mutation (data not shown), although several reports suggested that recombinant viruses belonging to the genus Enterovirus were generated by intra/interspecies transmission [52, 53]. Point mutations in the viral genome are common among picornaviruses because their polymerase lacks the proofreading ability and fidelity of amplification [54-56]. In addition, VP1 is a capsid protein, which likely influences host immunity in infected animals [35]. Therefore, the accumulation of mutations in the viral genome and subsequent selection by immunity in infected hosts may result in the generation of novel species.

Although the complete ORF and complete or partial UTRs sequences of four Japanese BEVs were determined, the virus could not be isolated from one diarrheal feces of a calf (BEV-AN12). BEV-AN12 has mutations in VP1 and $2 \mathrm{~A}$, which are involved in the formation of the capsid protein-host receptor binding site and cell proliferation, respectively [35, 49]. Although critical motifs for their function including [PS] ALXAXETG and GXCG were identified in the BEV-AN12 genome, a short insertion $(6 \mathrm{aa})$ in the $2 \mathrm{~A}$ protein region and non-synonymous substitution at the junction of VP3/VP1 were observed in the BEV-AN12 genome. Because these mutations may show alter receptor binding or virus replication, further crystal structure analysis of virions should be conducted.

The VP1 proteins in viruses belonging to the genus Enterovirus are widely known to components that form the receptor binding site (this site is referred to as the "canyon") together with VP2 and VP3 [31]. Reverse genetic analysis of other enteroviruses revealed that amino acid substitution in the VP1 region was responsible for the virus phenotype, such as pathogenicity and cell tropism [32-34]. Mutations in capsid protein genes may influence the structure of the "canyon" and receptorbinding capacity. BEVs also form a "canyon" on the outer side of the virion, although the cell surface receptor for BEVs is unknown. Therefore, BEVs may also have specific determinants for their phenotypes based on the aa sequences in the capsid protein encoding region. We also tried to investigate the prevalence of BEV-AN12, using VP1 specific primers, (Additional file 1: Table S1) in 38 diarrheal and 28 non-diarrheal feces samples collected from calves in Kagoshima prefecture during 2014-2015; feces from only one calf was positive, as revealed in the results. Therefore, we could not analyze the relationship between BEV-AN12 and its pathogenicity. According to the results of deep sequencing, we identified bovine picornavirus- and bovine kobu-like virus in the Kagoshima sample. There are reports 


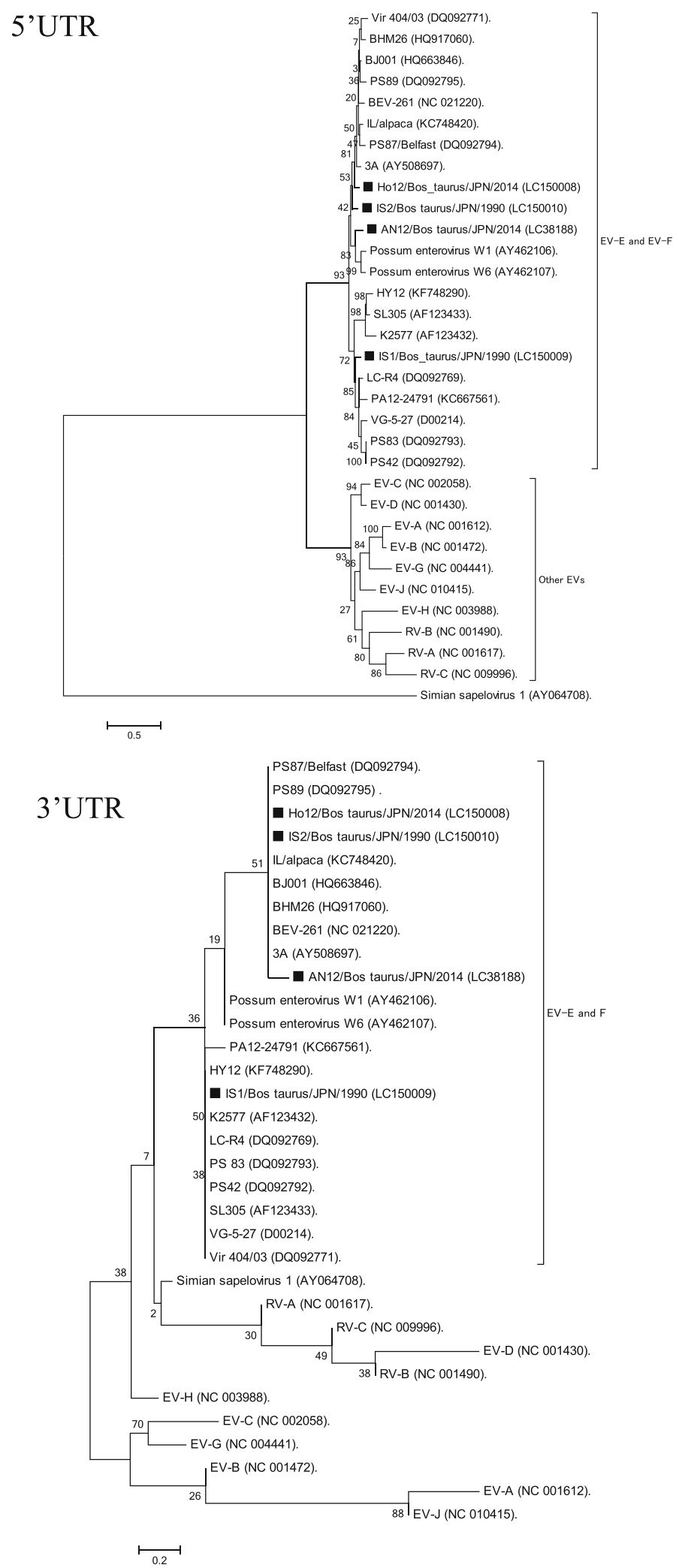

Fig. 2 Phylogenetic trees using nucleic acid sequences of $5^{\prime} U T R$ and $3^{\prime} U T R$. Phylogenetic trees were constructed using nucleic acid sequences of EV-A to EV-J and Japanese BEVs based on the maximum likelihood method in MEGA5.22 with bootstrap values calculated for 1000 replicates. Scale bar indicates nucleotide substitutions per site. BEVs in Japanese were indicated as $\mathbf{a}$. Simian sapelovirus (AY064708) was used as outgroup 


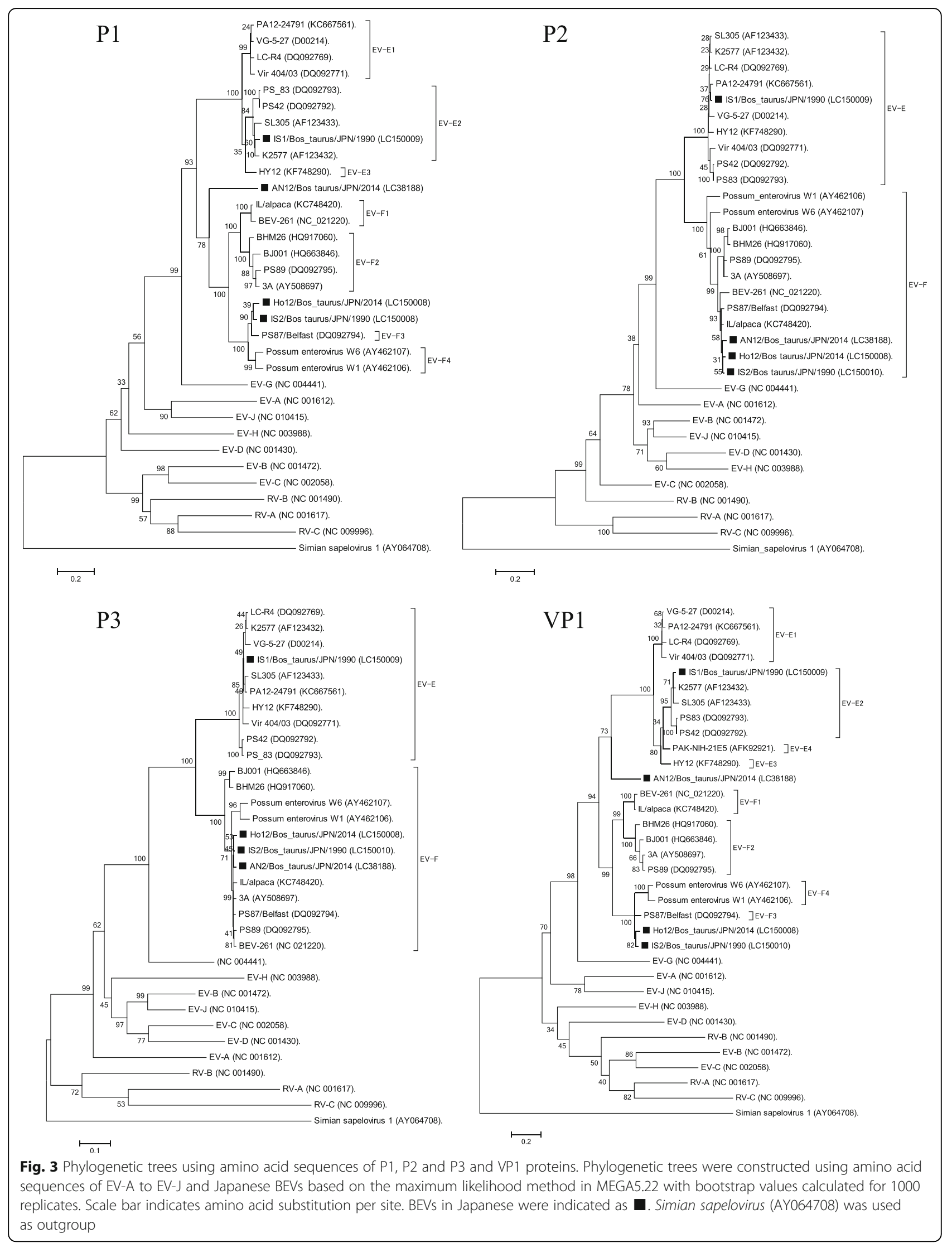


suggesting that bovine picornavirus and bovine kobuvirus are associated with diarrhea $[39,57]$. However, the pathogenicity of these viruses is still unknown. There is a possibility that all viruses can cause diarrhea. To clarify the determinants of the pathogenicity of BEVs, experimental infection based on reverse genetic analysis is necessary.

\section{Conclusions}

The present study identified novel BEV possessing highly divergent aa sequences in the VP1 coding region in Japan. We name this strain as "Enterovirus $K$ ", which is a novel species within the genus Enterovirus. To exclude the pathogenicity of BEVs, further genomic information must be accumulated.

\section{Additional files}

Additional file 1: Table S1. Primers information for VP1 sequencing. (PDF $84 \mathrm{~kb}$ )

Additional file 2: Table S2. Multiple alignments result using amino acid sequences of polyprotein. (PDF $458 \mathrm{~kb}$ )

\section{Abbreviations}

aa: Amino acid; BEV: Bovine enterovirus; EV: Enterovirus; IRES: Internal ribosome entry site; nt: Nucleotide; ORF: Open reading frame; UTR: Untranslated region

\section{Acknowledgements}

Not applicable.

\section{Funding}

This study was supported by the Research Project for Improving Food Safety and Animal Health of the Ministry of Agriculture, Forestry and Fisheries of Japan.

\section{Availability of data and materials}

Deep sequencing results and Japanese BEVs genomic sequences have been deposited in the DNA Data Bank of Japan (DRA005341, DRA005342, DRA005343, DRA005344, LC038188, LC150008, LC150009 and LC150010). All data related to phylogenetic trees have been deposited in TreeBASE (http://purl.org/phylo/treebase/phylows/study/TB2:S20312).

\section{Authors' contributions}

ST performed almost all in vitro experiments, analyzed and interpreted the data. In addition, ST wrote the manuscript. SS-R performed VP1 genome sequencing. KO contributed to collect the sample from Kagoshima prefecture in Japan, interpreted the data clinically and reviewed the manuscript. HA contributed to collect the sample from Hokkaido in Japan, interpreted the data including phylogenetic analysis and reviewed the manuscript. MK was a contributor to the molecular experiment and interpretation of the data including secondary RNA structure analysis of the $5^{\prime}$ untranslated region. YN was a contributor in revising the manuscript. TO was a major contributor in writing and revising manuscript. KS was a contributor in revising the manuscript. SO-T was a contributor in revising the manuscript. YK was a major contributor to the construction of CDNA library for deep sequencing. MO was a contributor to PCR experiment. MN coordinated and designed this study. In addition, MN was a major contributor to the interpretation of deep sequencing analysis. TM provided the funds necessary for the project, provided guidance to the study design. In addition, TM was a major contributor in writing and reviewing the manuscript, and is the corresponding author. All authors read and approved the final manuscript.

\section{Competing interests}

The authors declare that they have no competing interests.
Consent for publication

Not applicable.

Ethics approval and consent to participate

This section is not applicable because the viral isolates from a cow were not originally obtained for the purpose of this study.

\section{Author details}

${ }^{1}$ The United Graduate School of Veterinary Sciences, Gifu University, 1-1 Yanagito, Gifu-shi, Gifu 501-1193, Japan. ${ }^{2}$ Research and Education Center for Prevention of Global Infectious Disease of Animals, Tokyo University of Agriculture and Technology, 3-5-8 Saiwai-cho, Fuchu-shi, Tokyo 183-8509, Japan. ${ }^{3}$ Joint Faculty of Veterinary Medicine, Kagoshima University, 1-21-24 Korimoto, Kagoshima-shi, Kagoshima 890-0065, Japan. ${ }^{4}$ Faculty of Veterinary Science, Nippon Veterinary and Life Science University, 1-7-1 Kyonan-cho, Musashino-shi, Tokyo 180-8602, Japan. ${ }^{5}$ Faculty of Bioresources and Environmental Sciences, Ishikawa prefectural University, 1-308, Suematsu, Nonoichi-shi, Ishikawa 921-8836, Japan.

Received: 22 August 2016 Accepted: 28 December 2016

Published online: 17 January 2017

\section{References}

1. Hyypia T, Hovi T, Knowles NJ, Stanway G. Classification of enteroviruses based on molecular and biological properties. J Gen Virol. 1997;78:1-11.

2. King AM, Adams M, Carsten E, Lefkowitz E. Virus Taxonomy. Ninth Report of the International Committee for the Taxonomy of Viruses. San Diego: Academic; 2012.

3. Zell R, Sidigi K, Henke A, Schmidt-Brauns J, Hoey E, Martin S, et al. Functional features of the bovine enterovirus $5^{\prime}$-non-translated region. J Gen Virol. 1999;80:2299-309.

4. Zell R, Krumbholz A, Dauber M, Hoey E, Wutzler P. Molecular-based reclassification of the bovine enteroviruses. J Gen Virol. 2006;87:375-85.

5. Zell R, Stelzner A. Application of genome sequence information to the classification of bovine enteroviruses: the importance of $5^{\prime}$ - and 3'-nontranslated regions. Virus Res. 1997:51:213-29.

6. Kunin CM, Minuse E. New bovine viral agents isolated in Michigan. Public Health Repts. 1957;72:251.

7. Kunin CM, Minuse E. The Isolation in Tissue Culture, Chick Embryo and Suckling Mice of Filtrable Agents from Healthy Dairy Cattle. J Immunol. 1958:80:1-11.

8. Moll T, Finlayson AV. Isolation of cytopathogenic viral agent from feces of cattle. Science. 1957;126:401-2.

9. Nollens HH, Rivera R, Palacios G, Wellehan JFX, Saliki JT, Caseltine SL, et al. New recognition of Enterovirus infections in bottlenose dolphins (Tursiops truncatus). Vet Microbiol. 2009;139:170-5.

10. McClenahan SD, Scherba G, Borst L, Fredrickson RL, Krause PR, Uhlenhaut C. Discovery of a bovine enterovirus in alpaca. PLoS One. 2013:8:e68777.

11. Zheng T. Characterisation of two enteroviruses isolated from Australian brushtail possums (Trichosurus vulpecula) in New Zealand. Arch Virol. 2007;152:191-8.

12. Woo PCY, Lau SKP, Li T, Jose S, Yip CCY, Huang Y, et al. A novel dromedary camel enterovirus in the family Picornaviridae from dromedaries in the Middle East. J Gen Virol. 2015;96:1723-31.

13. Knowles NJ, Barnett ITR. A serological classification of bovine enteroviruses. Arch Virol. 1985;83:141-55.

14. Dunne HW, Huang CM, Lin WJ. Bovine enteroviruses in the calf: an attempt at serologic, biologic, and pathologic classification. J. Am. Vet. Med. Assoc. 1974:164(3):290-4

15. Huck RA, Cartwright SF. Isolation and Classification of Viruses from Cattle During Outbreaks of Mucosal or Respiratory Disease and from Herds with Reproductive Disorders. J Comp Pathol Ther. 1964;74:346-65.

16. Barya MA, Moll T, Mattson DE. Antigenic analysis of bovine enteroviruses through studies of the kinetics of neutralization. Am J Vet Res. 1967;28:1283-94.

17. Shaukat S, Angez M, Alam MM, Sharif S, Khurshid A, Malik F, et al. Molecular identification and characterization of a new type of bovine enterovirus. Appl Environ Microbiol. 2012;78:4497-500.

18. Zhang H, Liu H, Bao J, Guo Y, Peng T, Zhou P, et al. Characterization of an Enterovirus species $E$ isolated from naturally infected bovine in China. Virus Res. 2014;191:101-7. 
19. Ang LW, Koh BK, Chan KP, Chua LT, James L, Goh KT. Epidemiology and control of hand, foot and mouth disease in Singapore, 2001-2007. Ann Acad Med Singapore. 2009;38:106-12.

20. Zou X, Zhang X, Wang B, Qiu Y. Etiologic and epidemiologic analysis of hand, foot, and mouth disease in Guangzhou city: a review of 4,753 cases. Braz J Infect Dis Off Publ Braz Soc Infect Dis. 2012;16:457-65.

21. Gear JH. Nonpolio causes of polio-like paralytic syndromes. Rev Infect Dis. 1984;6 Suppl 2:S379-384.

22. Huang H-I, Shih S-R. Neurotropic Enterovirus Infections in the Central Nervous System. Viruses. 2015;7:6051-66.

23. Zell R, Dauber M, Krumbholz A, Henke A, Birch-Hirschfeld E, Stelzner A, et al Porcine Teschoviruses Comprise at Least Eleven Distinct Serotypes: Molecular and Evolutionary Aspects. J Virol. 2001;75:1620-31.

24. Knight-Jones TJD, Rushton J. The economic impacts of foot and mouth disease - what are they, how big are they and where do they occur? Prev Vet Med. 2013;112:161-73

25. Dunne HW, Ajinkya SM, Bubash GR, Griel LC. Parainfluenza-3 and bovine enteroviruses as possible important causative factors in bovine abortion. Am J Vet Res. 1973:34:1121-6.

26. Weldon SL, Blue JL, Wooley RE, Lukert PD. Isolation of picornavirus from feces and semen from an infertile bull. J Am Vet Med Assoc. 1979;174:168-9.

27. Zhu L, Xing Z, Gai X, Li S, San Z, Wang X. Identification of a novel enterovirus $E$ isolates $\mathrm{HY} 12$ from cattle with severe respiratory and enteric diseases. PLoS One. 2014;9:e97730.

28. Ley V, Higgins J, Fayer R. Bovine Enteroviruses as Indicators of Fecal Contamination. Appl Environ Microbiol. 2002;68:3455-61.

29. Jiménez-Clavero MA, Escribano-Romero E, Mansilla C, Gómez N, Córdoba L, Roblas N, et al. Survey of Bovine Enterovirus in Biological and Environmental Samples by a Highly Sensitive Real-Time Reverse Transcription-PCR. Appl Environ Microbiol. 2005;71:3536-43.

30. Blas-Machado U, Saliki JT, Sánchez S, Brown CC, Zhang J, Keys D, et al. Pathogenesis of a Bovine Enterovirus-1 Isolate in Experimentally Infected Calves. Vet Pathol Online. 2011;48:1075-84.

31. Hogle JM, Chow M, Filman DJ. Three-dimensional structure of poliovirus at 2.9 A resolution. Science. 1985;229:1358-65.

32. Kataoka C, Suzuki T, Kotani O, Iwata-Yoshikawa N, Nagata N, Ami Y, et al. The Role of VP1 Amino Acid Residue 145 of Enterovirus 71 in Viral Fitness and Pathogenesis in a Cynomolgus Monkey Model. PLoS Pathog. 2015;11:e1005033.

33. Caine EA, Moncla LH, Ronderos MD, Friedrich TC, Osorio JE. A Single Mutation in the VP1 of Enterovirus 71 is Responsible for Increased Virulence and Neurotropism in Adult Interferon Deficient Mice. J Virol. 2016;90(19):8592-604.

34. Lin J-Y, Shih S-R. Cell and tissue tropism of enterovirus 71 and other enteroviruses infections. J Biomed Sci. 2014;21:18.

35. Goens SD, Botero S, Zemla A, Zhou CE, Perdue ML. Bovine enterovirus 2 complete genomic sequence and molecular modelling of a reference strain and a wild-type isolate from endemically infected US cattle. J Gen Virol. 2004:85:3195-203.

36. Schlottau K, Schulze C, Bilk S, Hanke D, Höper D, Beer M, et al. Detection of a Novel Bovine Astrovirus in a Cow with Encephalitis. Transbound Emerg Dis. 2016;63:253-9.

37. Reuter G, Boros Á, Pál J, Kapusinszky B, Delwart E, Pankovics P. Detection and genome analysis of a novel (dima) rhabdovirus (Riverside virus) from Ochlerotatus sp. mosquitoes in Central Europe. Infect Genet Evol J Mol Epidemiol Evol Genet Infect Dis. 2016:39:336-41.

38. Omatsu T, Tsuchiaka S, Hirata T, Shiroma Y, Okazaki S, Katayama Y, et al. Detection of enterovirus genome sequence from diarrheal feces of goat. Virus Genes. 2014;48:550-2.

39. Nagai M, Omatsu T, Aoki H, Kaku Y, Belsham GJ, Haga K, et al. Identification and complete genome analysis of a novel bovine picornavirus in Japan. Virus Res. 2015;210:205-12.

40. Nagai M, Omatsu T, Aoki H, Otomaru K, Uto T, Koizumi M, et al. Full genome analysis of bovine astrovirus from fecal samples of cattle in Japan: identification of possible interspecies transmission of bovine astrovirus. Arch Virol. 2015;160:2491-501

41. Naoi Y, Kishimoto M, Masuda T, Ito M, Tsuchiaka S, Sano K, et al. Characterization and phylogenetic analysis of a novel picornavirus from swine feces in Japan. Arch Virol. 2016;161(6):1685-90.

42. Otomaru K, Naoi Y, Haga K, Omatsu T, Uto T, Koizumi M, et al. Detection of novel kobu-like viruses in Japanese black cattle in Japan. J Vet Med Sci Jpn Soc Vet Sci. 2016;78(2):321-4
43. Blom N, Hansen J, Blaas D, Brunak S. Cleavage site analysis in picornaviral polyproteins: discovering cellular targets by neural networks. Protein Sci. 1996;5:2203-16

44. Tamura K, Peterson D, Peterson N, Stecher G, Nei M, Kumar S. MEGA5: molecular evolutionary genetics analysis using maximum likelihood evolutionary distance, and maximum parsimony methods. Mol Biol Evol. 2011:28:2731-9.

45. Zuker M. Mfold web server for nucleic acid folding and hybridization prediction. Nucleic Acids Res. 2003;31:3406-15.

46. Tsuchiaka S, Masuda T, Sugimura S, Kobayashi S, Komatsu N, Nagai M, et al. Development of a novel detection system for microbes from bovine diarrhea by real-time PCR. J Vet Med Sci Jpn Soc Vet Sci. 2016;78:383-9.

47. Kamer $\mathrm{G}$, Argos P. Primary structural comparison of RNA-dependent polymerases from plant, animal and bacterial viruses. Nucleic Acids Res. 1984;12:7269-82.

48. Chow M, Newman JFE, Filman D, Hogle JM, Rowlands DJ, Brown F. Myristylation of picornavirus capsid protein VP4 and its structural significance. Nature. 1987;327:482-6.

49. Hughes PJ, Stanway G. The $2 A$ proteins of three diverse picornaviruses are related to each other and to the $\mathrm{H}$-rev107 family of proteins involved in the control of cell proliferation. J Gen Virol. 2000;81:201-7.

50. Gorbalenya AE, Koonin EV, Donchenko AP, Blinov VM. Two related superfamilies of putative helicases involved in replication, recombination, repair and expression of DNA and RNA genomes. Nucleic Acids Res. 1989;17:4713-30.

51. Martínez-Salas E, Francisco-Velilla R, Fernandez-Chamorro J, Lozano G, Diaz-Toledano R. Picornavirus IRES elements: RNA structure and host protein interactions. Virus Res. 2015;206:62-73.

52. Zhang G, Haydon DT, Knowles NJ, McCauley JW. Molecular evolution of swine vesicular disease virus. J Gen Virol. 1999;80:639-51.

53. Boros Á, Pankovics P, Knowles NJ, Reuter G. Natural interspecies recombinant bovine/porcine enterovirus in sheep. J Gen Virol. 2012;93:1941-51.

54. Ward CD, Stokes MA, Flanegan JB. Direct measurement of the poliovirus RNA polymerase error frequency in vitro. J Virol. 1988;62:558-62.

55. Ferrer-Orta C, Arias A, Pérez-Luque R, Escarmís C, Domingo E, Verdaguer N Sequential structures provide insights into the fidelity of RNA replication. Proc Natl Acad Sci U S A. 2007:104:9463-8.

56. Ward CD, Flanegan JB. Determination of the poliovirus RNA polymerase error frequency at eight sites in the viral genome. J Virol. 1992;66:3784-93.

57. Reuter $\mathrm{G}$, Boros A, Pankovics P. Kobuviruses - a comprehensive review. Rev Med Virol. 2011;21:32-41.

\section{Submit your next manuscript to BioMed Central and we will help you at every step:}

- We accept pre-submission inquiries

- Our selector tool helps you to find the most relevant journal

- We provide round the clock customer support

- Convenient online submission

- Thorough peer review

- Inclusion in PubMed and all major indexing services

- Maximum visibility for your research

Submit your manuscript at www.biomedcentral.com/submit

) Biomed Central 